\title{
Study on Storage Characteristic of Navel Orange Based on ANN
}

\author{
Junfang Xia and Runwen $\mathrm{Hu}$ \\ College of Engineering and Technology Huazhong Agricultural University, \\ Wuhan 430070 China
}

\begin{abstract}
In order to predict storage life of navel orange, The model for the variable regularity of total soluble sugar, total acidity, vitamin $\mathrm{C}$, soluble solids, the sugar-acidity ratio in navel orange according to storage time was established based on BP artificial neural network. The results show that the multi-factor BP artificial neural network model has better predicted effect than single-factor one. When the number of the hidden layer neuron is 8 , the multi-factor BP artificial neural network model of total soluble sugar, total acidity, vitamin $\mathrm{C}$, soluble solids, the sugar-acidity ratio according to storage time was the most accurate, the correlation coefficient $\mathrm{R}$ between prediction and true value of storage time reached 0.98 , the prediction and true value of the model was 0.99 . As a result, the multi -factor BP artificial neural network model could be used to predict the navel orange storage life.
\end{abstract}

Keywords: Navel orange; storage life; BP artificial neural network.

\section{Introduction}

The quality of navel orange such as total soluble sugar, total acidity,VC,soluble solids, the sugar-acidity ratio will change along with storage. Grasping this regulation, finding out notable quality index related to storage and establishing prediction model about quality can provide the theoretical basis for predicting storage life of navel orange, control internal quality effectively, prevent deterioration of fruit, preserve the value of navel orange and make appropriate market decision in time. Variation of orange quality is nonlinear according to the time of storage, and the traditional linear regression model is difficult to build such a nonlinear system. Artificial neural network method is to simulate the thinking way of human being based on the working principle of human brain cells, so it can fully approach any complicated nonlinear relationship, and has powerful potential to solve the system control of highly nonlinear and serious uncertainty ${ }^{[1-6]}$.

In order to establish artificial neural network model, Rumelhert et al presented BP Algorithm Used in Multilayer Neural Network ${ }^{[7]}$, BP artificial neural networks are mostly applied in artificial neural network, The algorithm got very extensive application in nonlinear regression, Most of studies are about the BP artificial neural network ${ }^{[8]}$. For example, Liu Jianxue et $\mathrm{al}^{[9]}$ used BP neural network method to establish protein 
prediction model of different types and sizes of rice samples, investigated the prediction ability of the model, and reported that there were good correlation (with the correlation coefficients above 0.9) between the predictive value and the value of chemical analysis using traditional methods. Lin Ming,Zhao Chen,Liu Xuesong,Bai Yingkui,Tang Yanfeng,Yang Nanlin et al determined the contents of corn, Cordyceps amino acids, Amino Acids in Cordyceps Sinensis,vanilla activity, VC Yinqiao Tablets and Anthraquinone in Rhubarb by the method of Artificial Neural Network- near-infrared spectroscopy. The results showed that the method was an effective and practical method of non-linear correction method, and the standard deviation of their forecasts are better than the processing results by principal component regression and partial least squares regression linear models ${ }^{[10-15]}$.

In this study, the variety model of quality of navel orange along with storage time is based on artificial neural network, which helps to forecast the storage time and storage life by the change of quality of navel orange.

\section{Materials and Methods}

\subsection{Materials}

The test samples are 200 mature navel oranges picked randomly from many trees in Dingnan in Jiangxi province in December 7, 2007, encapsulated by plastic film Bag, refrigerated in the artificial climate box with the temperature of $5^{\circ} \mathrm{C}$ and humidity of $70 \%$. At interval of 10 days, randomly choosed 10 navel oranges were determined total soluble sugar, total acidity, vitamin $\mathrm{C}$, soluble solids and the sugar-acidity ratio, the calculated average value and the storage time(day) were uesd as input vector $\mathrm{P}$ and output vector $\mathrm{T}$ of neural network respectivly. 160 of the 200 samples were used as training set and 40 as validation set.

\subsection{Chemical Analysis}

The measurement of the soluble solids content in navel oranges was carried out by WYT-1 handhold saccharimeter; the contents of total soluble sugar were determined by phenol sulfuric acid method, the total acid contents were detected by aid-base titration method, and the method of 2,6-dichlorophenol indophenol was used to measure the contents of vitaminC.

\subsection{Determination of Storage Life}

The observation of the stored orange showed that Mildew spots appeared on the skin of a small number of orange samples at 170 days. At 180 days, black spots appeared on the skin of about $20 \%$ of the navel oranges, this phenomenon indicated that the navel oranges had been deteriorated and could not be used as food. Therefore, the critical value of storage life of navel orange should be 170 days. This article will find index of internal quality related with the storage time significantly and estabilish the correlation 
model of characteristic of navel oranges to storage time so as to predict the storage time of navel orange. the continued storage time could be got by critical value of storage time subtracts the storaged time, that is storage time.

\subsection{Structure of BP Artificial Neural Network}

The multi-player feed-forward neural network of directional transfer algorithm was applied to BP artificial neural network which contained input layer, hidden layer and output layer. In this study, both of the structural input layer and output layer of BP artificial neural network model for quality characteristics of navel orange to storage time (single factor) are unit-neuron. Input layer neuron is the internal quality index, the output layer neuron is the storage time. As shown in Fig.1,The input layer of BP artificial neural network model of multi-factor quality of navel orange according to storage time is 5 neurons, output layer is single neuron,as shown in Fig.2.

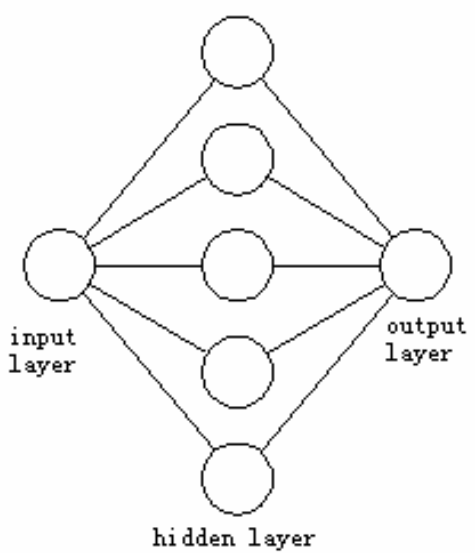

hidden layer

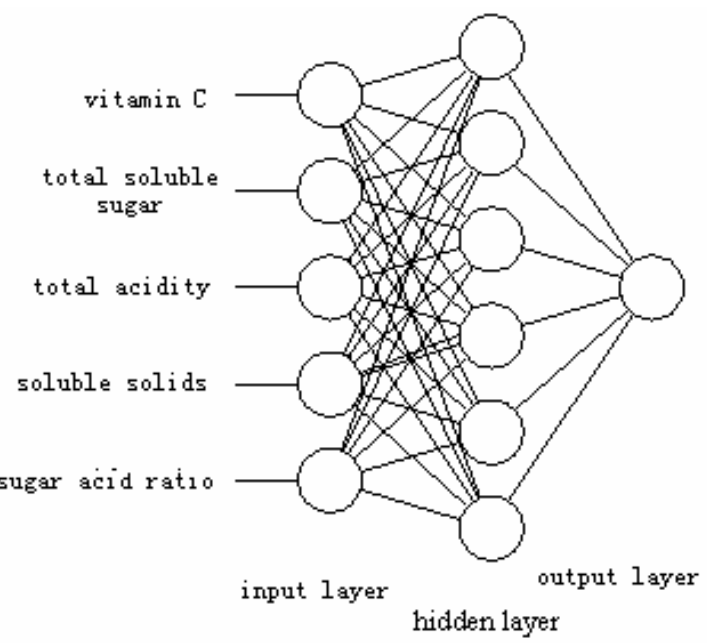

Fig. 1. The BP artificial neural network of single Fig. 2. The BP artificial neural network of factor multi factor

\subsection{Network Training Parameter}

In this study, Levenberg-Marquardt algorithm was used. S-transfer function was adopted to hidden layer neurons, the transfer function was tansig function; The output layer was cell neuron, linear transfer function was used in neuronal function and trainlm function was used in training function to generate the neural network of initialization. Before the training, the sample must be ascertained and should includ input $\mathrm{P}$ and corresponding output $\mathrm{T}$. During the training procedure, weight and threshold should be adjusted constantly to make the average variance mas of neural networks output and expection output vector to be the minimum. The definition of network training 
parameters: the max training times epochs was set as 200; the training iterative process show as 100; this training required the accuracy as 1e-10; learningrate $1 \mathrm{r}$ was demanded 0.01 ; the min gradient as $1 \mathrm{e}-6$; the max failure times must be under $5^{[7]}$.

\section{Data Processing and Analysis}

\subsection{Establishment of Single-Factor Artificial Neural Network Model}

Internal quality indexes such as total soluble sugar content, total acidity, vitamin $\mathrm{C}$, soluble solids and the sugar-acidity ratio of navel orange was taken as the input vector $\mathrm{P}$ and the storage time as expected output vector $\mathrm{T}$ of neural network. According to the programming and neural network training of definite network training parameters in the software MATLAB6.5, the quality to storage time of single-factor BP artificial neural network model for navel orange was established.

The number of hidden layer neuron was initiativly set as 5 with the method of trial and error. Begainning with smaller neuron,we trained and inspected the capability of internet. As the number of neuron increased gradually, the capability of internet was trained and inspected again untill the the related coefficient Rc of output and expect value of artificial neural network model reached the ideal value. Right now, the number of the neuron of hidden layer is perfect.

The characteristics of total soluble sugar content, total acidity, vitamin C, soluble solids and the sugar-acidity ratio of the 40 untraining samples were put into the neural network model established to predict the storage time. the conservasion of the established model will be analysed through comparing with true value.

The best hidden layer in the BP artificial neural network model for total soluble sugar content, total acidity, vitamin $\mathrm{C}$, soluble solids, the sugar-acidity ratio of navel orange according to storage time using was in table 1 as well as the effect on prediction and the proven results. In table $1, \mathrm{~N}$ is the number of best hidden layer, Rc is the related coefficient of predicted value of calibration model and measured value, $\mathrm{Rp}$ is the related coefficient of predicted value of validation model and measured value, $\mathrm{Wp}$ is the relative error of predicted value of the validation model and measured value.

Table 1. Predictive results of the single factor BP artificial neural network model

\begin{tabular}{ccccc}
\hline & $\mathrm{N}$ & $\mathrm{R}_{\mathrm{c}}$ & $\mathrm{R}_{\mathrm{p}}$ & $\mathrm{W}_{\mathrm{p}}(\%)$ \\
\hline Total soluble sugar & 60 & 0.864 & 0.88 & 7.34 \\
Total acidity & 50 & 0.984 & 0.9814 & 0.84 \\
Vitamin C & 50 & 0.82 & 0.8648 & 7.99 \\
Soluble solids & 30 & 0.933 & 0.9343 & 7.32 \\
Sugar acid ratio & 60 & 0.89 & 0.9 & 9.01 \\
\hline
\end{tabular}

The model's training research indicated that the number of neura in hidden layer of artificial neural network model had much influence to model's accuracy and was accordng to different quality index of hidden layer of artificial neural network model. 
The model's training time is related to the number of hidden layer neuron, the more of the number of hidden layer neuron,the longer of the training time; Among all model trainings, when the training time epochs was about 20,the mean square error of output layer reached the target error that training parameters demanded,and kept stable, not changed with epochs's increasing.

In the single-factor BP artificial neural network mode of navel orange's internal qualities, total soluble sugar, total acidity, vitamin $\mathrm{C}$, soluble solids and the sugar-acidity ratio according to storage time in BP artificial neural network mode were respectivly $60,50,50,30,60$.

The prediction accuracy and stability of the navel orange's internal qualities to storage time in BP artificial neural network model with signal factor from high to low in sequence were total acidity BP artificial neural network model $\rightarrow$ soluble solids BP artificial neural network model $\rightarrow$ sugar-acid ratio BP artificial neural network model $\rightarrow$ total soluble sugar BP artificial neural network model $\rightarrow$ BP artificial neural network model of vitamin $\mathrm{C}$. The result showed that the most obvious criterion of quality related to storage time in signal factor is total acidity. When the quantity of hidden layer neuron is 50,the total acidity to storage time of BP artificial neural network model accuracy is perfect,the related coefficient Rc can reach 0.984, the related coefficient $\mathrm{Rp}$ of validation model's predicted value and measured value is 0.9814 ,the average value of relative error is $0.84 \%$, the predictive effect is much better, so the neural network model established is stable, and can satisfy the prediction requirment of navel orange's storage life.

\subsection{The Establishment of the Multi-factor BP Artificial Neural Network}

The five indexes such as total soluble sugar content, total acidity, vitamin $\mathrm{C}$, soluble solids, sugar-acid ratio of navel orange are taken as input vector $\mathrm{P}$ of neuron network, the number of input layer neuron is 5 and the storage time is used as expected output vector $\mathrm{T}$. According to the definite network training parameters, programming and network training in the software MATLAB6.5, we established the multi-factor BP artificial neural network model. The method of trial and error was adopted to ascertain the optimum value of the hidden layer neuron's quantity. After input the multi-factor indiex of the 40 navel oranges' internal qualities untraining into the established neural network model and predict its storage time, the stability of the established model was analysed according to the comparison with measured value.

The reaserch of model training indicates that the multi-factor BP artificial neural network dynamic model by the 5 internal qualities of total soluble sugar content, total acidity, vitamin $\mathrm{C}$, soluble solids, sugar-acid ratio of navel orange according to the storage time is established,when the number of hidden layer neuron is 8 , the model accuracy is much high, the correlation coefficient Rc reach 0.98, the training time is shorter, the correlation coefficient of validation model's predicted value and measured value $\mathrm{Rp}$ is 0.99 , the average value of relative error is $6.701 \%$, predictive effect is much better,the accuracy and stability of the established neural network model is better than single factor model which indicates that multi-factor changes can better reflect the variation of navel orange's internal qualities to the storage time.using the multi -factor 
$\mathrm{BP}$ artificial neural network model to forecast the storage time and storage life of navel orange is appropriate.

\section{Conclusion}

The variety model of total soluble sugar, total acidity, vitamin C, soluble solids, the ratio of total soluble sugar to total acidity in navel orange according to storage time was established based on BP artificial neural network.. In the training of BP artificial neural network model, through changing the quantity of hidden layer neuron, the optimized number of the hidden layer neurons of the internal qualities of navel orange of BP artificial neural network with single-factor and five multi-factor was $\mathrm{N}$, and verify the established model.The research results as follows.

(1) The best forecast accuracy and stability of navel quality to storage time in single-factor BP artificial neural network model is total acidity of BP artificial neural network model, Therefore,in the single factor ,the index of quality which highlightly related to storage time is the total acidity of navel orange.And when the quantity of hidden layer neuron is 50,the accuracy of total acidity and storage time's BP artificial neural network model is much higher, the correlation coefficient Rc reach 0.984 ,the correlation coefficient Rp of validation model's predicted value and measured value is 0.9814 ,the average value of relative error is $0.84 \%$.

(2) When the number of hidden layer neuron is 8 , the BP artificial neural network dynamic model which based on the five quality characteristies of total soluble sugar, total acidity, vitamin $\mathrm{C}$, soluble solids and sugar acid ratio has the best prediction. The correlation coefficient Rc can reach 0.98 , correlation coefficient Rp of validation model's predicted value and measured value is 0.99 , the average value of relative error is $6.701 \%$.

(3) The association between multi-factor changes and storage time is most notable,the predictive validity and stability of multi-factor BP artificial neural network model along with storage time is better than single-factor model. it indicates that multi-factor changing effects can best reflect navel orange's qualities' variety. In the practical application, multi-factor BP artificial neural network model can be used to predict the storage time and storage life of navel orange.

\section{References}

[1] Guolin, W., Congming, H.: Application of neural network ensemble based on contractive algorithm in NIR analysis. Chinese Journal of Spectroscopy Laboratory 22(3), 473-476 (2005)

[2] Yanbin, W.: Application of artificial neural network in NIR analysis and Dark-colored oil analysis. Research Institute of Petroleum Processing A Dissertation for PH.D (August 2000) (in Chinese)

[3] Yong, Y., Ming, Q., Yunhong, L., et al.: Identification of alcohol quality via neural network based on genetic algorithms. Transactions of The Chinese Society of Agricultural Machinery 34(6), 104-106 (2003) (in Chinese) 
[4] Guangjun, Z.: Applications of artificial neural network to opto-electric measurements. Journal of Beijing University of Aeronautics and Astronautics 27(5), 554-568 (2001) (in Chinese)

[5] Changhong, D., Matla, B.: Applications of neural network. National Defence Industry Publishing, Beijing (2005) (in Chinese)

[6] Fuqiang, L., Wencui, Z., Ge, L., et al.: Nondestructive quality control of rutin pharmaceuticals by near infrared reflectance spectroscopy and artificial neural network. Chemical Analysis and Meterage 12(3), 11-13 (2003) (in Chinese)

[7] Fei Sike Technology R \& D Center. MATLAB 6.5 Neural Network Analysis and Design of Auxiliary. Electronics Publishing, Beijing (2005) (in Chinese)

[8] Xiaoming, Q., Luda, Z., Xiaolin, D., et al.: Quantitative analysis using NIR by building PLS-BP model. Spectroscopy and Spectral Analysis 23(5), 870-872 (2003) (in Chinese)

[9] Jianxue, L., Shouyi, W., Ruming, F.: Determination of protein content of rice by near infrared spectroscopy based on neural networks. Journal of Jiangsu University 25(3), 196-198 (2004) (in Chinese)

[10] Ming, L., Jin, L.: Determination on components of corns based on neural networks and near infrared spectrum. Infrared Technology 26(3), 78-81 (2004) (in Chinese)

[11] Chen, Z., Haibin, Q., Yiyu, C.: A new approach to the fast measurement of content of amino acids in cordyceps sinensis by ANN-NIR. Spectroscopy and Spectral Analysis 24(1), 50-53 (2004) (in Chinese)

[12] Xuesong, L., Haibin, Q., Yiyu, C.: Determination of Active Components in a Natural Herb with Near Infrared Spectroscopy Based on Artificial Neural Networks. Chem. Res. Chinese. U 21(1), 36-43 (2005)

[13] Bai, Y., Shen, X., Ding, D.: Two-component nondestructive analysis of VC Yinqiao tablets with NIR and bp neural network. Laser \& Infrared 34(5), 354-356 (2004) (in Chinese)

[14] Yanfeng, T., Zhuoyong, Z., Guoqiang, F.: Identification of official rhubarb samples based on NIR spectra and neural networks. Spectroscopy and Spectral Analysis 24(11), 1348-1351 (2004) (in Chinese)

[15] Nanlin, Y., Yiyu, C., Haibin, Q.: Quantitative determination of mannitol in cordyceps sinensis using near infrared spectroscopy and artificial neural networks. Chinese Journal of Analytical Chemistry 31(6), 664-668 (2003) (in Chinese) 